\title{
Examination of a novel double diabetes model
}

\author{
György Eigner*, Balázs Kurtán *, Imre J. Rudas ${ }^{\dagger}$, Chui Chee Kong ${ }^{\ddagger}$ \\ and Levente A. Kovács* \\ *Physiological Controls Group, Obuda University, Budapest, Hungary \\ Email: eigner.gyorgy@phd.uni-obuda.hu, [kovacs.levente, kurtan.balazs]@nik.uni-obuda.hu \\ ${ }^{\dagger}$ University Research and Innovation Center, Obuda University, Budapest, Hungary \\ Email: rudas@uni-obuda.hu \\ ${ }^{\ddagger}$ Control and Mechatronics, Department of Mechanical Engineering, National University of Singapore, Singapore \\ Email: mpecck@nus.edu.sg
}

\begin{abstract}
In this paper we investigate a novel diabetes model able to model both type 1 and 2 diabetes mellitus and beyond this it could handle mixed state of these diseases (Double Diabetes) by DDEs (Delay Differential Equation). Through different investigations we analyze this model, its behavior and its parameters' sensitivity. Further, it is compared with a known diabetes model by different complementing digestion models.
\end{abstract}

\section{INTRODUCTION}

Modeling constitutes an essential part in control engineering [1], [2], as well in the research of diabetes [3]. Because of the complexity of the human metabolic system, intense abstraction is needed. To reduce this complexity, the modeling tasks can be divided into four main groups:

1) Patient models, describing the behavior of a diabetic patient (type 1, type 2, double, or other kind of diabetes);

2) Digestion models, as inputs of diabetes models responsible for the modeling of the entire absorption (through the digestion) - complex models (intakes are complex nutrition) or reduced models (intakes are carbohydrates (CHO) or glucose);

3) Sensor models, modeling the blood sugar sampling method or the continuous glucose measuring (CGM) process;

4) Other modeling tasks like noise simulation, disturbances, machine models, etc.

In this paper we concentrate on the first two groups (patient models and digestion models), and try out different meal intake protocols to analyze the behavior of the selected models. All used patient models are associated directly or indirectly with the Minimal Model [4]. This was the first model successfully used to simulate the changing of the plasma glucose level of a diabetic patient. Since then, several patient models have been developed [3], [5], [6], classifying them in two main parts:

- Low complexity models with few states (usually 3-5), concentrating on the glucose-insulin dynamics;

- High complexity models with several states (more then 5) that preferably gives extended description of the metabolic system.
The main area of use for the low complexity models is the Intensive Care Units (ICU). Here the purpose is the realization of Tight Glycemic Control (TGC) protocol - to hold the patient's blood sugar level in a given range [7]-[9]. On the other side, the high complexity models are used to describe the accurate behavior of the diabetic patient. Generally, these models are highly nonlinear [10]-[14]. Classification of the digestion models can be done similarly to patient models:

- Reduced models, where in most of the cases the nutrient intake represents glucose bolus and the absorption function contains slowly decreasing exponential part completed with the biological specifics of the glucose [10], [15];

- Complex models capable simulating the process of the digestion and absorption, wherein the nutrient intakes contain mixed meals [14], [16]-[18].

The paper is composed as follows: first, we present and investigate a novel diabetes model appeared in the literature. Secondly, we compare the model behavior with a well-known high complexity diabetes model. Third, we perform simulations of the model supplemented with reduced and complex digestion models. Conclusion and future work end the current article.

\section{ANALYSIS OF THE NOVEL DOUBLE DIABETES MODEL}

The model in purpose was published in 2013 [15] and contains the following equations:

$$
\begin{aligned}
& \dot{G}_{p}(t)=G_{i n}(t)+H G P\left(I_{p}\left(t-\tau_{1}\right)\right)-U_{i i}\left(G_{p}(t)\right) \\
& -E\left(G_{p}(t)\right)-k_{1} G_{p}(t)+k_{2} G_{i}(t) \\
& \dot{G}_{i}(t)=k_{1} G_{p}(t)-k_{2} G_{i}(t)- \\
& -U_{i d}\left(G_{p}(t), Q_{1 a}(t), Q_{1 b}(t), Q_{2}(t)\right) \\
& \dot{Q}_{1 a}(t)=p u(t)-L D_{a}\left(Q_{1 a}(t)\right)-k_{a 1} Q_{1 a}(t) \\
& \dot{Q}_{1 b}(t)=(1-p) u(t)-L D_{b}\left(Q_{1 b}(t)\right)-k_{a 2} Q_{1 b}(t) \\
& \dot{Q}_{2}(t)=k_{a 1} Q_{1 a}(t)-k_{a 1} Q_{2}(t) \\
& \dot{I}_{p}(t)=\alpha I S\left(G_{p}\left(t-\tau_{2}\right)\right)+k_{a 1} Q_{2}(t)+k_{a 2} Q_{1 b}(t)- \\
& -k_{e} I_{p}(t)
\end{aligned}
$$


It is composed from a type 1 diabetes model (T1DM), and a type 2 diabetes model (T2DM) handling the time delays. The model subparts combine ordinary differential equations (ODEs) with delay differential equations (DDEs) [15], where the state variables are: $G_{p}(t)$ and $G_{i}(t)$ the amount of glucose in plasma and subcutaneous (SC) compartments [mg], $Q_{1 a}(t)$, $Q_{1 b}(t)$ and $Q_{2}(t)$ insulin masses in the SC compartment [mU] and $I_{p}(t)$ insulin mass in the plasma [mU].

$u(t)$ represents the injected insulin flow [ $\mathrm{mU} / \mathrm{min}$ ] and is the input of the system, while $G_{i n}(t)$ is the glucose intake obtained from the meal absorption $[\mathrm{mg} / \mathrm{min}] . \frac{G_{i}(t)}{V_{G}}$ can be considered the output of the model (here this is the glucose concentration) in SC (accessible) compartment. The summarized properties of this model can be found in [15].

The visual formulation of the state dependency of the model is depicted in Fig. 1.a.

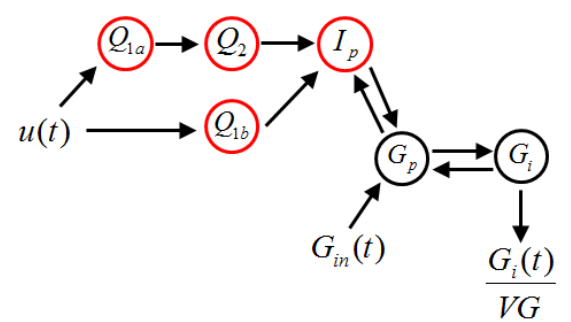

(a)

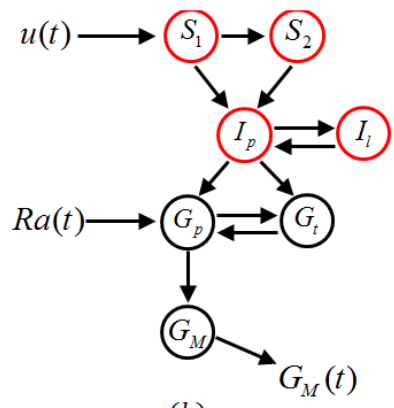

(b)

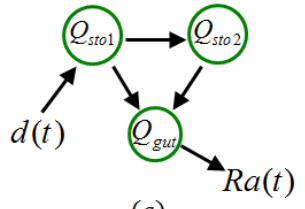

(c)

Figure 1. The schematic structure of the compared models [(a) novel double diabetes model, (b) Magni model, (c) Dalla Man model]

\section{A. Controllability and Observability}

The model contains two explicit delays, namely an Insulin Secretion Delay (ISD), and a Hepatic Glucose Production Delay (HGPD). An observation can be instantly made, the normoglycemic state is varying due to the parametric changes in the model (1). As a simplification, our assumption was that it is possible to eliminate the effect of the delays if and only if the analysis is done near to the steady-states.
In our analysis, we have developed a symbolic solver in order to obtain the critical points, with zero rate of change for each state (relying on the current parameters of the model). The default parameter set was used from [15], along with Case 11 patient's parameters (32 years old, $82 \mathrm{~kg}$ T1DM male patient data) of [15]. After obtaining the critical states, the results (i.e. the normoglycemic states) were substituted in the differential equations to check, if they represent a critical point or not. Afterward, the physiologically relevant steady-states were determined, and the model was linearized in that working point, considering no inputs (zero insulin and $\mathrm{CHO}$ intake).

The linearized model's controllability and observability matrices proved that the system have full rank for both.

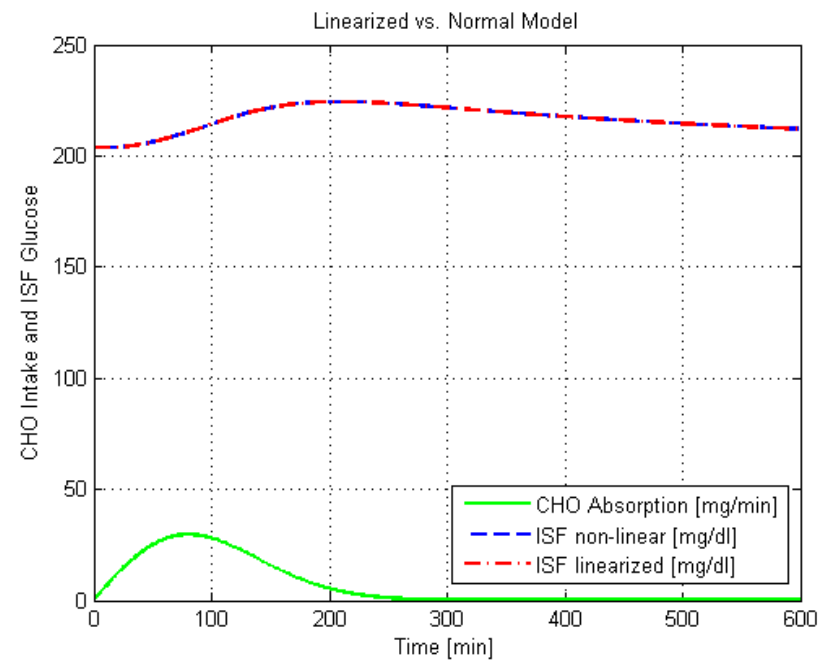

Figure 2. Linearized model vs. nonlinear ISF glucose

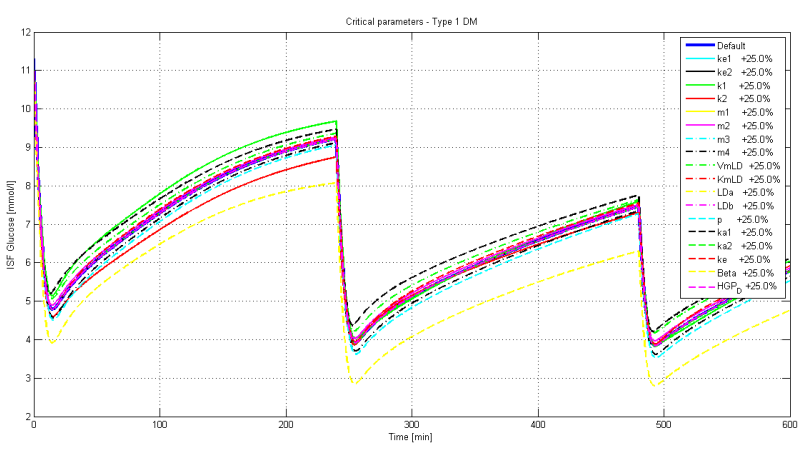

Figure 3. T1DM model parameter sensitivity analysis results

The state-space representation of the linearized model had negative real eigenvalues indicating that the model is asymptotically stable at and near to the working point. This observation has an important physiological aspect: when the material exchange is stable between the compartments, the state variables are changing in order to remain around the steady-state values considering the long-term evaluation of the system. This means the maintenance of homeostasis. However, if the 
biological parameters (hence, the metabolism) is impaired, the states can change outside of the normal boundaries, resulting in hypo- or hypoglycemic states.

Fig. 2. shows the similarity between the original nonlinear and the linearized system response for arbitrary carbohydrate intake and zero insulin administration in T1DM case.

\section{B. Parameter Sensitivity Analysis. TIDM Case}

By parameter sensitivity analysis it is possible to investigate the most sensitive parameters of the system. A classification regarding the impairment of the patient can be made in two different categories.

At the beginning, the T1DM occurrence will be examined (with insulin injections). In this case the $\alpha$ parameter is considered zero (i.e. the pancreas does not excretes insulin), thus the ISD is zero too. Parameter perturbations were made for all relevant parameters with $+25 \%$ of their original values.

The considered meal intake [g] was converted into glucose absorption [ $\mathrm{mg} / \mathrm{min}$ ] based on [15]. In this case, the equivalent of a $45 \mathrm{~g} \mathrm{CHO}$ intake (at zero time of the analysis), plus a 4 Unit of short acting insulin were injected. Fig. 3. shows the result of the sensitivity analysis, where the initial states were obtained with our symbolic solver on Case 11 patient of [15]. $k_{1}, k_{2}$ and $\beta$ were identified as the most sensitive parameters for the T1DM case in the short term, while if insulin therapy is applied, then on the long term $m_{4}, p, k_{a 1}$ resulted as the most sensitive ones. The paper focuses on the short term parameter investigation, because in a real life case short term accuracy is much more desirable to be obtained for adequate control; furthermore, the states can be adjusted to follow the measurement data from the patient.

1) Critical Parameter Analysis for the T1DM Case: Parameter $k_{1}, k_{2}$ are glucose transfer rate parameters, modeling material exchange between the plasma and the subcutaneous region. The physiological interpretation reveals the inner glucose exchange that depends on the diabetic type. In the short term $\beta$ represents the insulin resistance parameter. This parameter implies greater or lesser insulin dependent glucose utilization. In the analysis we used 5\% perturbation steps, from $-30 \%$ to $+30 \%$.

One can see that the $\beta$ parameter has a significant effect on the boundaries of the glucose level. This problem is known as 'double diabetes' for T1DM patients [19]. Fig. 4. and Fig. 5. shows how the $k_{1}$ and $k_{2}$ effects the evaluation of the glucose concentration in the subcutaneous region, while Fig. 6. demonstrates how the insulin resistance 'offsets' the glucose amount, when insulin is administered.

\section{Parameter Sensitivity Analysis. T2DM Case}

For the T2DM occurrence the parameters were assigned from [15] as well: Case 20 of [15] was used (70 years old, $52.7 \mathrm{~kg}$

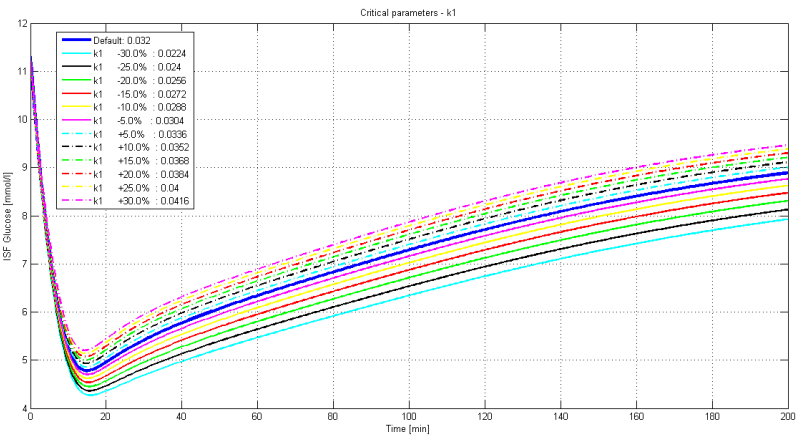

Figure 4. T1DM $k_{1}$ parameter's effect

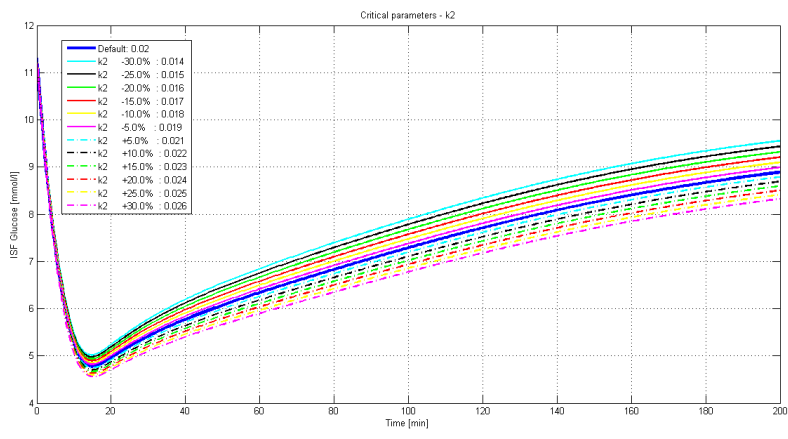

Figure 5. T1DM $k_{2}$ parameter's effect

T2DM male patient data), with 37 min considered ISD and 45 min of HGPD. $\alpha$ was $0.42, \beta$ was $0.52, k_{a 1}$ was 0.14 and $k_{a 2}$ was 0.13 , while the other parameters remained unchanged. The results of the sensitivity investigation are presented in Fig. 7.

For the T2DM case the results revealed that $k_{1}, k_{2}, k_{e}, \alpha, \beta$, $H G P D$, and $I S D$ were the critical parameters.

1) Critical Parameter Analysis for the T2DM Case: As for the T2DM case, the following parameters were investigated: $H G P D, I S D, k_{e}$, and $\alpha$. (The $k_{1}, k_{2}, k_{e}$, and $\beta$ parameters were investigated in the T1DM case.) Fig. 8. shows how the HGPD changes the hepatic glucose production. Higher delays results in greater amplitude that could imply fatal problems, when glucose level drops to hypoglycemia.

Fig. 9. demonstrates the effect of ISD, which can greatly amplify the oscillations, for glucose both in the plasma and interstitial regions. The impairment of the patient's metabolism is represented by the two delays.

Fig. 10. represents the analysis of insulin clearance in plasma, caused by the liver, namely for parameter $k_{e}$. When decreasing it, the results will be in terms of higher plasma insulin.

Fig. 11. illustrates how the pancreas's insulin secretion rate changes the glucose levels in the interstitial region. Increasing $\alpha$ results in higher plasma insulin concentration. 


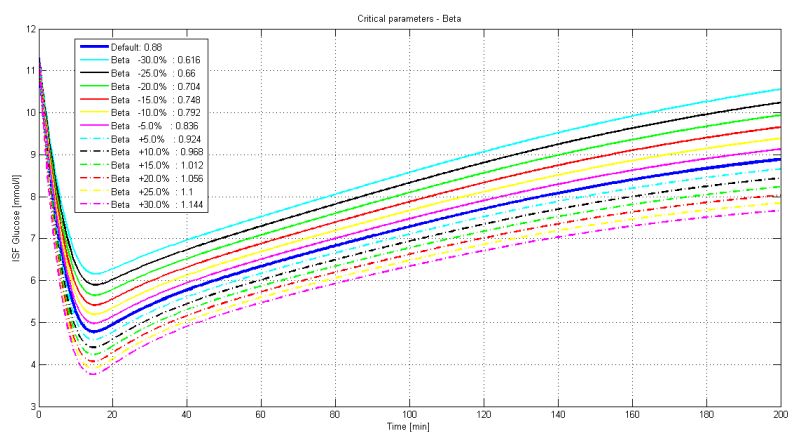

Figure 6. T1DM $\beta$ parameter's effect

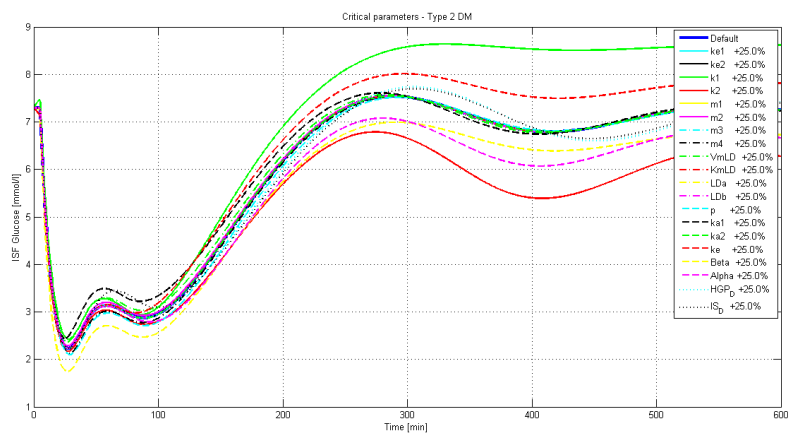

Figure 7. T2DM model parameter sensitivity analysis results

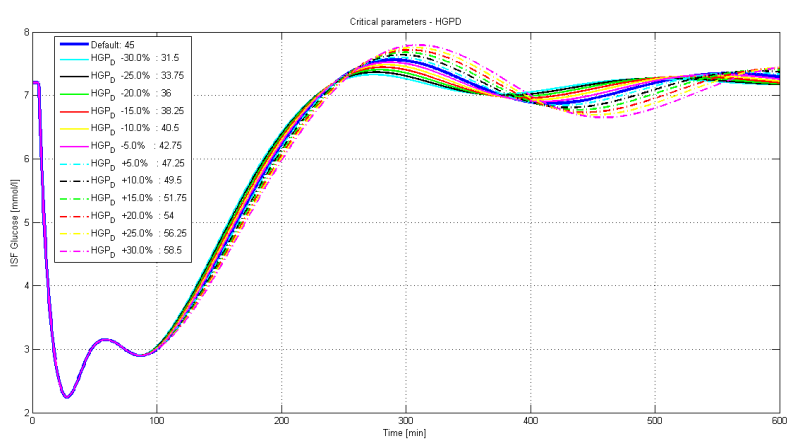

Figure 8. T2DM $H G P D$ parameter's effect

\section{Combined Effect of the Delays}

The T2DM results concluded that the two delays are significant from both physiological and modeling point of view. Hence, we have investigated the combined effect of the two delays. Results are presented in Fig. 12.

The results show that the amplitude of the glucose level oscillation is affected by the combination of both delays. This characteristics strengthens the idea that if the sum of the two delays are getting bigger (showing some kind of impairment), the glucose level limits are growing bigger as well.

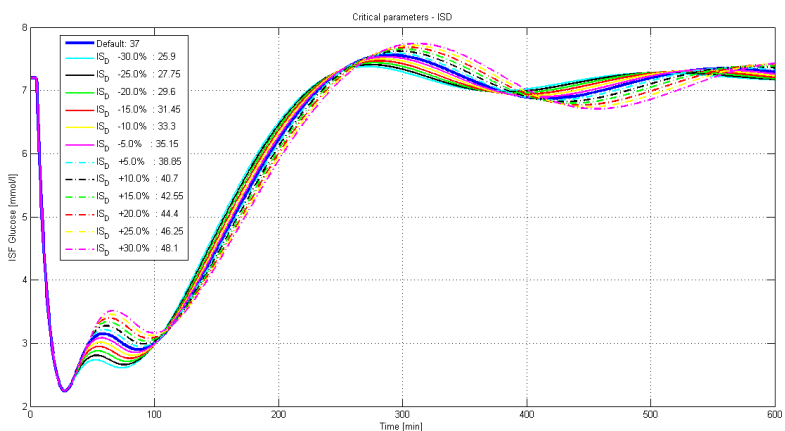

Figure 9. T2DM ISD parameter's effect

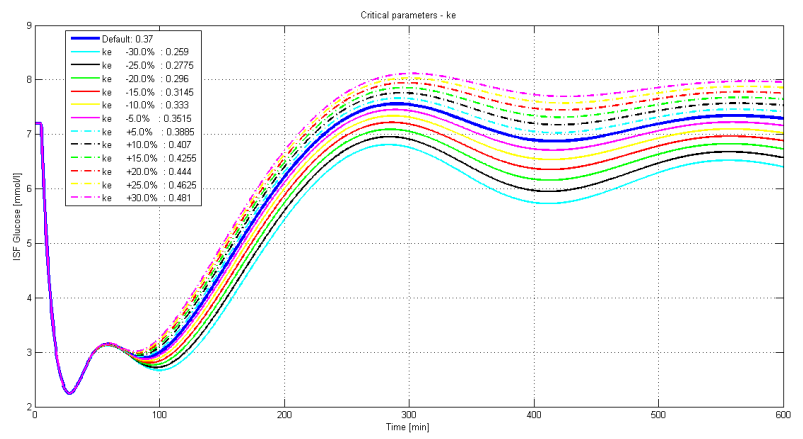

Figure 10. T2DM $k_{e}$ parameter's effect

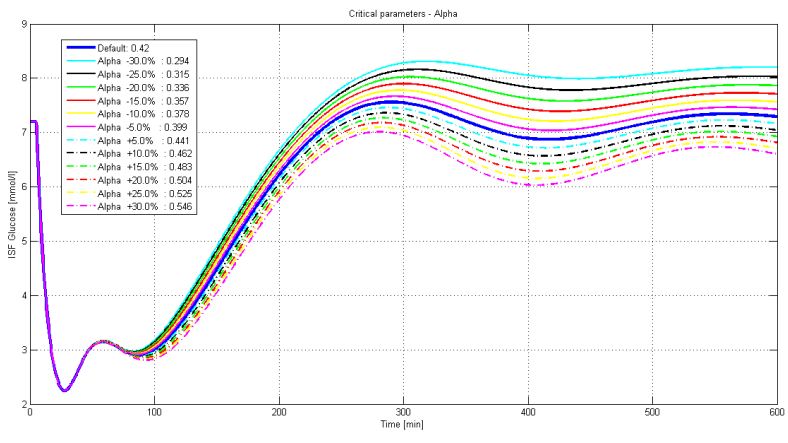

Figure 11. T2DM $\alpha$ parameter's effect

\section{COMPARISON OF DM MODELS}

In order to investigate the characteristics of the investigated diabetes model, we have performed a comparison with the well-known T1DM model of Magni et al. [20].

The idea of the 10th order high-complexity model of [20] is similar with the considered model of [15]. It contains two inputs, $R a(t)$ and $u(t)$ representing the meal $[\mathrm{mg} / \mathrm{kg} / \mathrm{min}]$ and insulin $[\mathrm{mU} / \mathrm{min}]$ inputs and one output, $G M(t)$ reflecting the subcutaneous glucose level [mg/dl]. The model is depicted on Fig. 1.b. The comparison of the two diabetes models confronted the following main problems: 


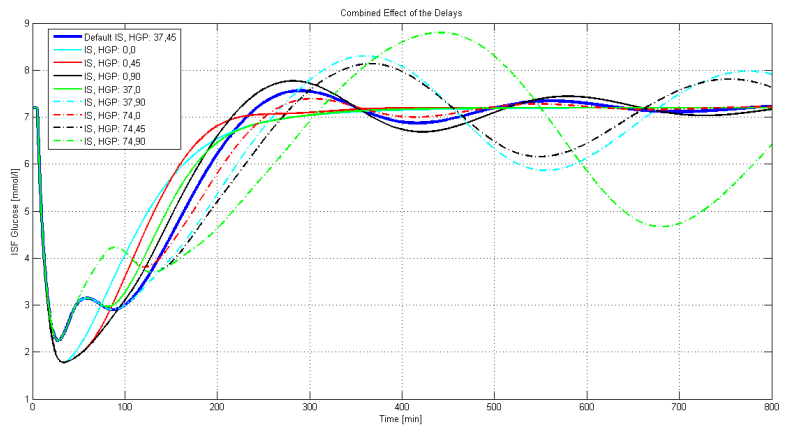

Figure 12. Combined effect of IS and HGP delays

- different number of states;

- the model of [15] contains time-delays instead of one of the [20] model;

- the subcutaneous levels are calculated differently.

\section{A. Description of the digest}

We used two digestion models (a reduced and a complex one) to complete the examinations. The reduced model has been introduced in [15], having the following equation:

$$
G_{m}\left(t_{m}, k, b\right)=\frac{k t e x p\left(-\left(t-t_{m}\right)^{2} / 2 b^{2}\right)}{b^{2}} * u\left(t-t_{m}\right)
$$

where $k$ and $b$ are parameters without dimension, $t m$ is the time moment of meal boluses and $u\left(t-t_{m}\right)$ is a unit step function with unity value [g], if $t>=t m$.

The complex model was taken from [20] and known in the literature as the "Dalla Man model". This model is based on 3 states and contains annexed exponential part too, depicted in Fig. 1.c. The model input is the meal bolus [g], while the output is the absorbed glucose [mg].

\section{B. Results of the comparisons}

We compared the models as follows: first, we declared different intake protocols, then select the same digestion model and execute the comparison. The protocols considered were the followings:

P1. 48 hours long simulations, with the input protocol given by [15] for case 1 , started from $5 \mathrm{mmol} / \mathrm{L}$;

P2. 48 hours long simulations, with the input protocol given by [15] for case 20 , started from $5 \mathrm{mmol} / \mathrm{L}$;

P3. 48 hours long simulations, without any inputs and started from $5 \mathrm{mmol} / \mathrm{L}$.

The results can be seen on Fig. 13., Fig. 14. and Fig. 15.

Fig. 13. shows the obtained results, with Reduced digestion model, beside P1 (upper) and P2 (lower) protocols. On Fig.
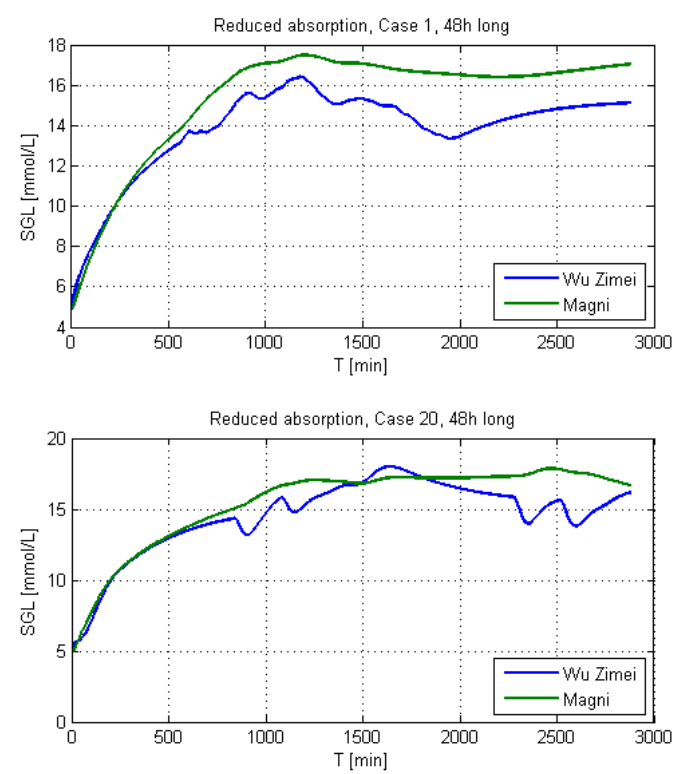

Figure 13. Results of open loop simulation with Reduced digestion model, SGL (Subcutaneous Glucose Level) [mmol/L]
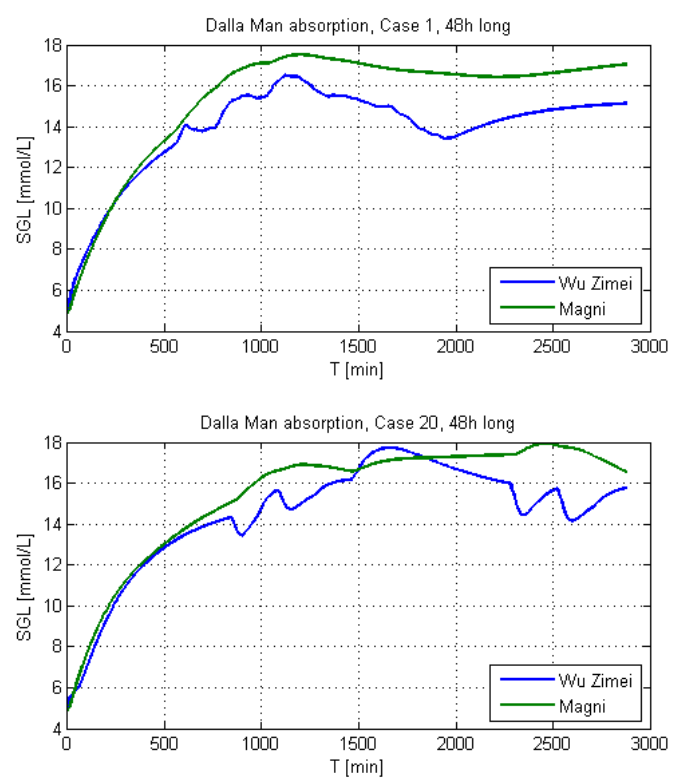

Figure 14. Results of open loop simulation with Dalla Man digestion model SGL (Subcutaneous Glucose Level) $[\mathrm{mmol} / \mathrm{L}]$

14. it can be seen the results for the P1 (upper) and P2 (lower) protocols using the "Dalla Man digestion model". Result of P3 protocol is depicted in Fig. 15. Fig. 16 shows the amount of absorbed glucose with Reduced (upper) and Dalla Man (lower) models. 


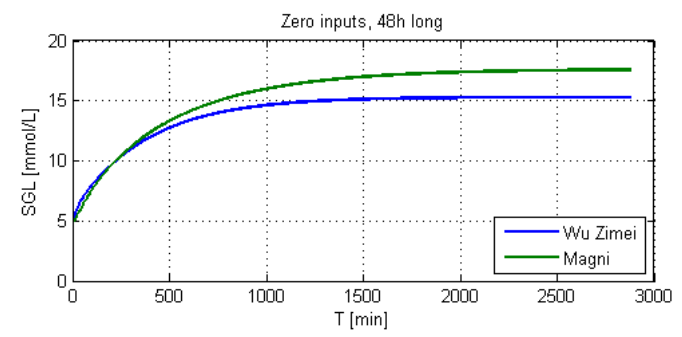

Figure 15. Results of open loop simulation with Zero inputs, SGL (Subcutaneous Glucose Level) $[\mathrm{mmol} / \mathrm{L}]$
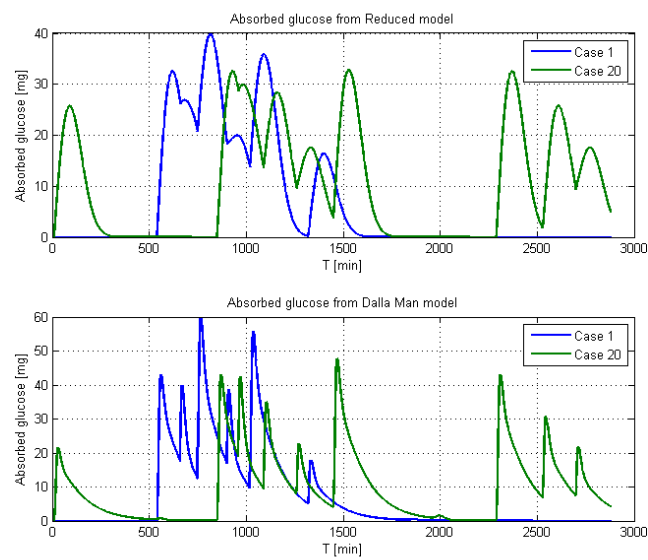

Figure 16. Characteristics of the absorption from Reduced and Dalla Man model

\section{CONCLUSION AND FUTURE WORK}

As the last figures suggest, the obtained results are quite similar, as expected, proving the validity of the investigated DM model.

As next step, we will expand the examination with other patient and digestion models and investigate the opportunity to involving other submodels, too. Furthermore, we are going to try out different identification and estimation possibilities on these complex systems.

\section{ACKNOWLEDGMENTS}

L. Kovács is supported by the János Bolyai Research Scholarship of the Hungarian Academy of Sciences.

\section{REFERENCES}

[1] W. Levine, Ed., The Control Engineering Handbook, 2nd ed. Boca Raton, FL, US: CRC Press, Taylor and Francis Group, 2011.

[2] J. Nyers, L. Garbai, and A. Nyers, "Analysis of heat pump condenser's performance using the mathematical model and a numerical method,' ACTA Pol Hun, vol. 11, no. 3, pp. 139-152, 2014.

[3] F. Chee and T. Fernando, Closed-Loop Control of Blood Glucose. Springer, 2007.
[4] G. Toffolo, R. Bergman, D. Finegood, C. Bowden, and C. Cobelli, "Quantitative estimation of beta cell sensitivity to glucose in the intact organism - a minimal model of insulin kinetics in the dog," Diab, vol. 29, pp. 979-990, 1980.

[5] C. Cobelli, E. Renard, and B. Kovatchev, "Artificial pancreas: Past, present and future," Diab, vol. 60, no. 11, pp. 2672-2682, 2011.

[6] V. Shah, A. Shoskes, B. Tawfik, and S. Garg, "Closed-loop system in the management of diabetes: Past, present, and future," Diab Techn \& Therap, vol. 16, no. 8, pp. 477-490, 2014.

[7] X. Wong, J. Chase, G. Shaw, C. Hanna, T. Lotz, J. Lin, I. Singh-Levett, L. Hollingsworth, O. Wong, and S. Andreassen, "Model predictive glycaemic regulation in critical illness using insulin and nutrition input: A pilot study," Med Eng \& Physics, vol. 28, pp. 665-681, 2006.

[8] T. Van Herpe, M. Espinoza, N. Haverbeke, B. Moor, and G. den Berghe, "Glycemia prediction in critically ill patients using an adaptive modeling approach,” J Diab Sci Techn, vol. 1, pp. 348-356, 2007.

[9] J. Dickson, J. Hewett, C. Gunn, A. Lynn, G. Shaw, and J. Chase, "On the problem of patient-specific endogenous glucose production in neonates on stochastic targeted glycemic control," J Diab Sci Techn, vol. 7, pp. 913-927, 2013.

[10] R. Hovorka, V. Canonico, L. Chassin, U. Haueter, M. Massi-Benedetti, M. Orsini-Federici, H. Pieber, T.R. Schaller, L. Schaupp, T. Vering, and M. Wilinska, "Nonlinear model predictive control of glucose concentration in subjects with type 1 diabetes," Physiol Meas, vol. 25, pp. 905-920, 2004.

[11] M. Wilinska, L. Chassin, C. Acerini, J. Allen, D. Dunger, and R. Hov orka, "Simulation environment to evaluate closed-loop insulin delivery systems in type 1 diabetes," J Diab Sci Techn, vol. 4, no. 1, pp. 132-144, 2010.

[12] J. Sorensen, "A physiological model of glucose metabolism in man and it use to design and access improved insulin therapies for diabetes,' Ph.D. dissertation, Department of Chmical Engineering, Massachusetts Institute of Technology, 1985.

[13] L. Magni, D. Raimondo, L. Bossi, C. Dalla Man, G. De Nicolao, B. Kovatchev, and C. Cobelli, "Model predictive control of type 1 diabetes: An in silico trial," J Diab Sci Techn, vol. 1, pp. 804-812, 2007.

[14] C. Dalla Man, R. Rizza, and C. Cobelli, "Meal simulation model of the glucose-insulin system," Trans Biomed Eng, vol. 54, no. 10, pp. $1740-1750,2007$

[15] W. Zimei, "Mathematical models with delays for glucose-insulin regulation and applications in artificial pancreas," Ph.D. dissertation, Doctor of Philosophy Department of Mechanical Engineering, National University of Singapore, 2013.

[16] C. Dalla Man, R. Rizza, and C. Cobelli, "Mixed meal simulation model of glucose-insulin system," in 28th IEEE EMBC Conference, New York, USA, 2006, pp. 307-310.

[17] C. Dalla Man, M. Camilleri, and C. Cobelli, "A system model of oral glucose absorption: Validation on gold standard data," Trans Biomed Eng, vol. 53, no. 12, pp. 2472-2479, 2006.

[18] T. Arleth, S. Andreassen, M. Orsini-Federici, A. Timi, and M. MassiBenedetti, "A model of glucose absorption from mixed meals," in 4th IFAC Modelling and Control Biomedical Systems, 2000, pp. 1-6.

[19] S. J. Cleland, B. M. Fisher, H. M. Colhoun, N. Sattar, and J. R. Petrie, "Insulin resistance in type 1 diabetes: what is 'double diabetes' and what are the risks?" Diabetologia, Springer, vol. 56, no. 7, pp. 1462-1470, 2013.

[20] L. Magni, D. Raimondo, C. Dalla Man, G. De Nicolao, B. Kovatchev, and C. Cobelli, "Model predictive control of glucose concentration in subjects with type 1 diabetes: an in silico trial," in 17th IFAC World Congress, Seoul, Korea, 2008, pp. 4246-4252. 